\title{
A Teaching Mode for Art Anatomy Based on Digital Virtual Technology
}

\author{
https://doi.org/10.3991/ijet.v13i08.9053 \\ Na Wang \\ University of Science and Technology Liaoning, Anshan, Liaoning \\ 3121383141 @qq. com
}

\begin{abstract}
Art anatomy is a morphological science characterized with the use of many terms and descriptions that are difficult to remember. The traditional art anatomy teaching mode pays attention to explanation of theoretical knowledge in the classroom using simple teaching methods, so that the teaching is boring. Consequently, students have low interest in learning, which affects the quality of teaching. With this regard, an Art Anatomy teaching mode was proposed in this study based on digital virtual technology. It uses digital virtual technology to build 3D models and database of art anatomy teaching specimens. Due to the powerful modeling functions and production effect of 3DS MAX, MAYA software and VRML to render realistic scenes, the preservation and recording of the initial state of the anatomical specimens can save various types of data and spatial relationships of the specimens to the greatest extent. Based on the teaching practice in the course of "Art Anatomy" at Liaoning University of Science and Technology in Liaoning Province, China, the teaching mode proposed in this paper was found to be highly efficient, concrete and easy to understand, and students had a high degree of recognition for this teaching method. A new idea and method were thereby provided for the teaching reform of art anatomy.
\end{abstract}

Keywords—-Teaching Mode, Digital Virtual Technology, Art Anatomy

\section{Introduction}

Anatomy is a crucial foundation course in medical, sports, and art academy teaching. Art anatomy is a branch of anatomy, and it is the foundation of art creation for students majoring in art. The study of art anatomy directly influences the learning quality of other foundation and major courses [1]. Art anatomy is the primary means to record human creation and civilization processes, and it embodies the direct function of art creation. Art anatomy is important for current art creation and teaching practice of art foundation.

To cultivate qualified art creation talents, art colleges set art anatomy as a professional required course. It is also a foundation course for freshmen. However, the teaching mode that combines textbooks, images, specimens, and models is often applied in traditional anatomy teaching. Human body specimen are lacking, and image 
teaching is not sufficiently vivid. These situations bring about many learning obstacles for beginners. Thus, the existing anatomy teaching mode and method need to be reformed. Problem-based learning (PBL) [2] is a learning method based on problems. In PBL, the teacher is the guide, and the students are the subjects. Students learn about problem solving and solve problems in learning. Digital virtual technology as the teaching means and PBL as the teaching method were applied in art anatomy teaching in this study, and an effect analysis was conducted by selecting art anatomy as the teaching case.

\section{State of the art}

Virtual reality (VR) is also called "virtual environment" [3]. After the US implemented the Visible Human Project (VHP), the United States National Library of Medicine announced its successful development of a male VHP anatomy dataset. It is the first to do so. With this dataset, many relevant application software were developed internationally. Kurillo [4] et al. used real-time stereoscopic vision and the virtual environment to display a remote immersion anatomy system. The system was used to capture users' 3D head portraits in real time and project them to the shared virtual environment so that patients and therapists can interact remotely. Such a remote system was proven to have a good practical effect. Faria et al. [5] proposed an anatomy software based on digital virtual technology and applied it to the treatment of pediatric inpatients. The system includes digital virtual teaching demonstration and applies a simulation scene of a high-fidelity anthropometric dummy. Experiments showed that the system has a high application value in clinical teaching. One of the digital virtual anatomy teaching methods currently used by Chinese hospitals is the standalone version of the Chinese Digital Human Anatomy System applied by Hangzhou Medical College [6]. Another example is the 3D digital visual human engineering of the United States National Library of Medicine introduced by Changchun University of Chinese Medicine. A fully digital virtual visual human 3D anatomical model is constructed on the platform, and the structure is vivid. Bones, muscles, fibrous connective tissues, blood vessels, and nerves form an obvious contrast. The platform can be used as an examination system and connected to a website to examine students' mastery of anatomy knowledge. The "3D virtual human anatomy display platform V2.0" applied by Guangzhou Medical University is yet another example. The platform includes image entry and image processing systems. Specimen images at different angles are captured at $360^{\circ}$ by a Nikon D7000 DSLR camera to obtain rectangular projection or cubic graphs of spherical panorama at different angles. The image processing system developed by J Builder 9.0 and Delphi is used for splicing to obtain images of the $3 \mathrm{D}$ virtual specimen. The images are classified and arranged according to each anatomy system. Text description is also provided, and all-around interactive viewing of real specimens at all directions is achieved. These digital virtual human bodies digitalize and visualize information on human biology; thus, they make quantitative analysis, calculation, and accurate simulation of computers possible and provide the foundation for the integration of digital technology and anatomy [7]. On the 
basis of this research, a few researchers combined digital virtual technology with the PBL teaching method and applied it to anatomy teaching. However, most students and teachers feel that a course with a strong theoretical property is dull and difficult to remember in the teaching process of art anatomy. For example, a course that involves numerous structure nouns of anatomy and tedious contents is considered dull by students. A positive learning atmosphere in the classroom is difficult to create, so the learning effect is poor [8].

Therefore, a new method based on digital virtual technology and PBL teaching was proposed and applied in art anatomy teaching. The method combines major and course characteristics. The key point of this method is that it designs problems in advance, motivates students to think, and makes them practice with problems. This method aims to cultivate students' self-learning ability, allow them to grasp knowledge on art anatomy, and lay a solid foundation for future major study under limited conditions.

\section{$3 \quad$ PBL art anatomy teaching mode based on digital virtual technology}

The traditional method of teaching art anatomy is based on classroom teaching and adopts a wall map, a model, a specimen, and the human body as supplementary instruction tools. Then, the specimen anatomy is examined to deepen students' understanding of classroom content. In recent years, various colleges in China exhibited a shortage of teaching specimens for different degrees, leading to a severe lack of anatomy learning and reduced teaching quality. Digital virtual technology teaching provides complete, continuous, and fine human 3D anatomic images. Moreover, 3D visual and virtual human anatomy models can be constructed. With such models, students can observe the human structure from any angle and any profile and can magnify, rotate and translate the structure to be observed. By carefully observing and clearly understanding the spatial relationship between each organ and tissue of humans, students can form an entire image of the human structure in their mind. The emergence of the digital virtual human lets students see the structure vividly and clearly, overcomes the problems of specimen shortage and non-repeated use of specimens in anatomy teaching, and reduces the teaching cost. The PBL teaching method is applied. Fig. 1 shows a relation graph of the PBL teaching method in digital art anatomy. 


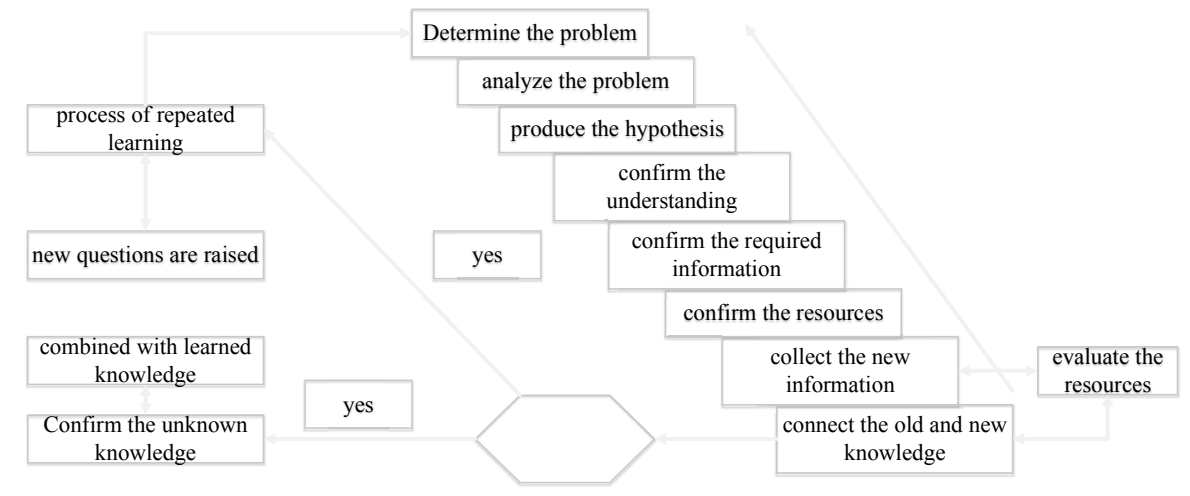

Fig. 1. Relation graph of the PBL teaching method in digital art anatomy

The teaching method mainly includes the following.

1. Problem development stage [9]: This stage involves two problems. The first one is the source of problems. The sources of problems are multiple, such as independent development by the teacher, joint development by the teacher and students, or development by students. The second one is the nature of problems. The problems in PBL should be complex, exploratory, and challenging with sufficient clues.

2. Problem inquiry stage [10]: This is an important scientific inquiry process that includes the following steps: define the problem, establish a hypothesis, combine known and new experiences, propose solving steps, gather data, analyze and exchange data, and put forward possible solutions. The process is shown in Fig. 1.

3. Problem verification stage: This stage includes verifying the learner's scheme, submitting a task report, and evaluating the learner's performance.

Collection and processing of anatomy specimen data are the foundation of digitization. Data accuracy and comprehensiveness restrict the digitization effect. Data texture processing also influences data quality.

Data collection. Common data collection methods include dot and plane approaches. The dot method [11] acquires the space coordinates and attributes of largesize paleontological fossil through conventional group measurement tools and methods, such as total station and GPS receiver. The plane method utilizes aviation and space remote sensing to obtain large-area image records. The specimen data collection process is as fig.2.

As shown in Fig. 2, a digital camera is first applied to acquire basic framework data of specimens. Camera specification, shooting order, and unity of number should be considered in the shooting process to avoid errors in data saving and to prepare for specimen modeling and $3 \mathrm{D}$ virtual scene generation. 


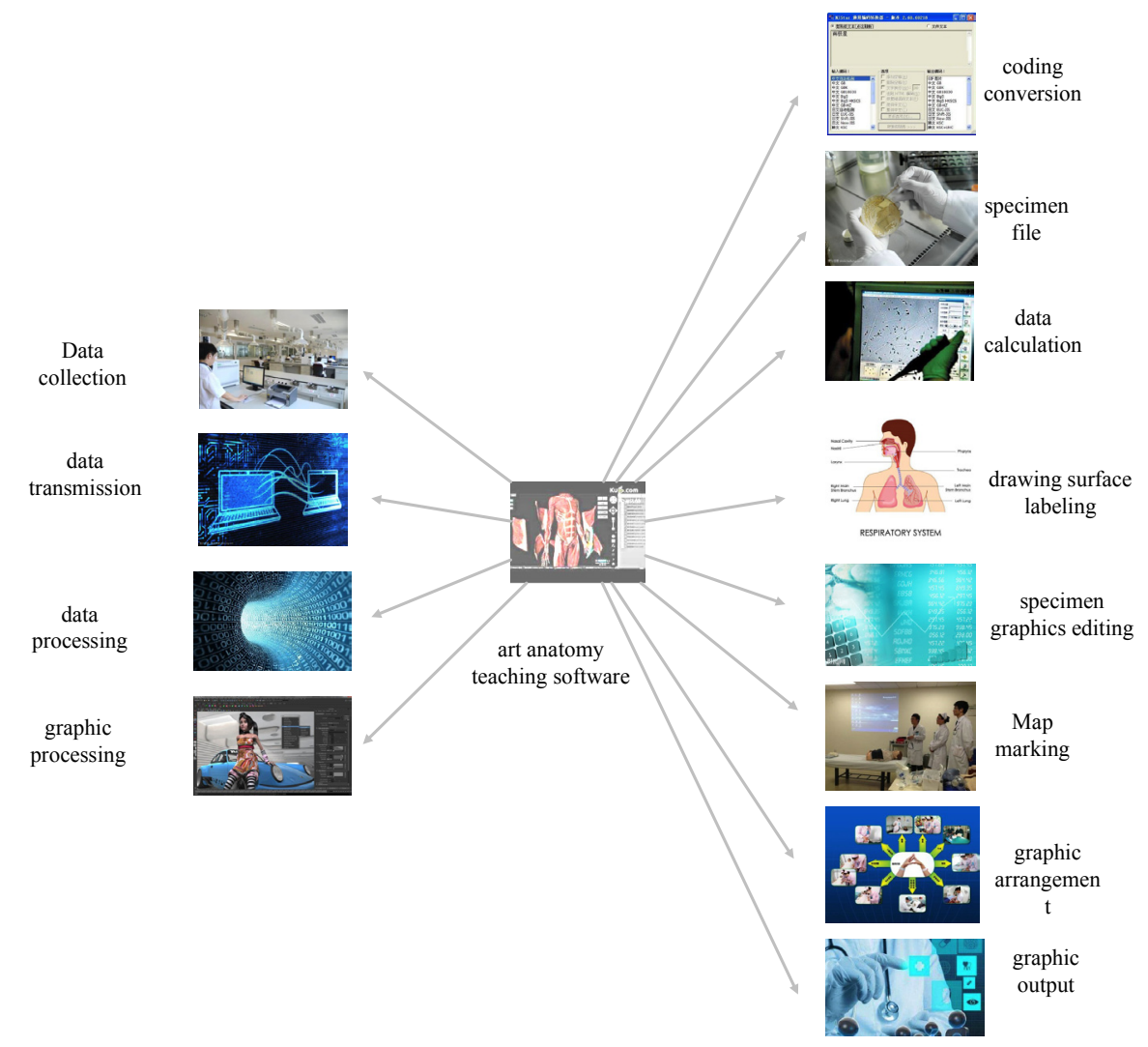

Fig. 2. Anatomy specimen data collection process

Second, a total station is used to gather data. Profile graph surveying and mapping of specimens should be paid attention to. Traditional profile graph surveying and mapping technology separates different profiles to make splicing incompleteness appear in the splicing process. The total station can be combined with the centering rod to confirm all profiles at one time. Finally, a computer is used to edit. The total station can accurately reflect $3 \mathrm{D}$ relations of specimens, and $3 \mathrm{D}$ coordinates can be obtained. In the end, they may be edited by using a computer to form graphs.

Data processing. Data processing is performed after data collection. Modeling, namely, large-size paleontological fossil modeling, is conducted according to the data collected. This modelling should be conducted according to the picture proportion to accurately show the cultural relics processed digitally. Next, modeling in 3DX MAX is performed. The primary model is established with the hierarchical modeling method. On this basis, the spatial position and size of the fossil structure are established according to pictures and measurement data. Finally, texture rendering is conducted. The pictures are processed in accordance with the browsers' visual effect. A shadow and perspective effect is also added to the digital model. 
Texture processing. Given that data collection was conducted using digital pictures, 2D mapping was selected in this research. The original data of the pictures are stored in the internal storage in the form of an array, which is called texture element in texture mapping. The array is regarded as a continuous 2D rectangular textured pattern $\mathrm{T}(\mathrm{s}, \mathrm{t})$. The two independent variables, $\mathrm{s}$ and $\mathrm{t}$, are called texture coordinates. The surface of the geometric object is expressed as $(\mathrm{u}, \mathrm{v})$, namely, the mapping from $(\mathrm{u}, \mathrm{v})$ to $(\mathrm{x}, \mathrm{y}, \mathrm{z})$ or $(\mathrm{x}, \mathrm{y}, \mathrm{z}, \mathrm{w}) .(\mathrm{x}, \mathrm{y}, \mathrm{z})$ or $(\mathrm{x}, \mathrm{y}, \mathrm{z}, \mathrm{w})$ represents the projection process of the geometric object from the physical coordinate system to the screen coordinate system. This process is abstracted as a mapping function of texture coordinated $\mathrm{s}$ and $t$. Texture element $\mathrm{T}(\mathrm{s}, \mathrm{t})$ corresponds to the color value of a position in the color cache, and it is expressed with the function

$$
\begin{aligned}
& \mathrm{x}_{s}=\mathrm{x}_{s}(s, t) \\
& \mathrm{y}_{s}=\mathrm{y}_{s}(s, t)
\end{aligned}
$$

where (xs, ys) represents a position in the color cache. For a geometric object whose surface is expressed with parameters, the texture mapping process is shown in Fig. 3.

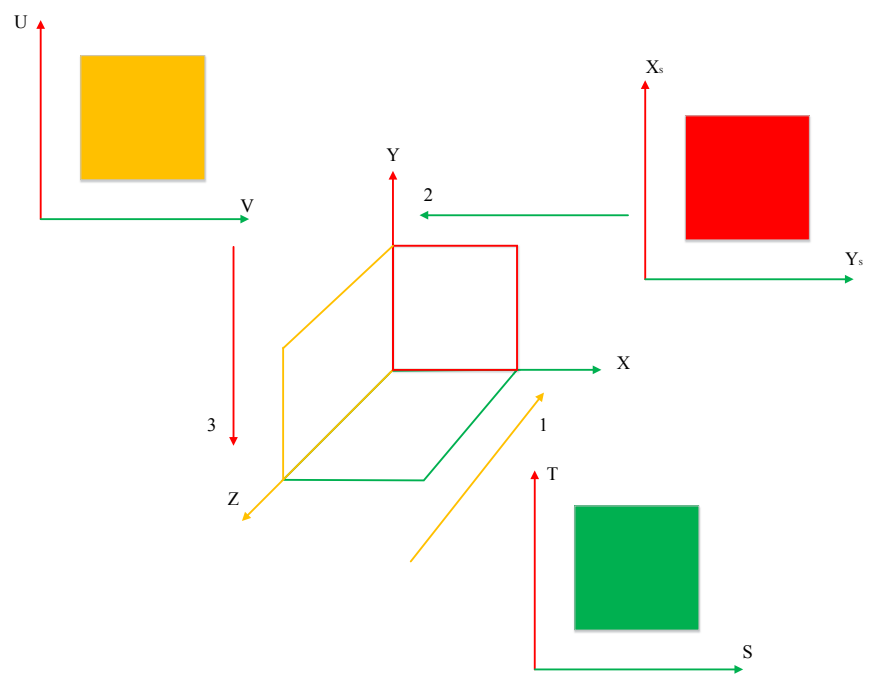

Fig. 3. Texture mapping process

Process 1 in Fig. 3 is the mapping from the texture coordinate system to the physical coordinate system. Process 2 is the mapping from the parameter coordinate system to the physical coordinate system. In Process 3, the object is transformed to the screen coordinate system from the physical coordinate system through a third mapping. The figure shows dot-to-dot mapping. Regional mapping is critical for the digital application of large-size paleontological fossil. Most dots on the surface can be expressed with parameters. $\mathrm{P}$ is a dot on the surface. The function expression is as formula 3: 


$$
P(u, v)=\left[\begin{array}{ll}
x & (u, v) \\
y & (u, v) \\
z & (u, v)
\end{array}\right]
$$

Under the precondition of known parametric surface, any texture element $\mathrm{T}(\mathrm{s}, \mathrm{t})$ in texture space can be mapped to a dot $\mathrm{P}(\mathrm{u}, \mathrm{v})$ on the surface through the following linear mapping relation:

$$
\begin{aligned}
& u=a s+b t+c \\
& v=d s+e t+f
\end{aligned}
$$

As long as ae $\neq \mathrm{bd}$, the mapping is reversible. In other words, the region can be mapped on the screen.

\section{Teaching Case and Teaching Effect}

Art anatomy was selected as the teaching example. Digital virtual technology and the PBL method were applied to create a teaching design for the course. Upon the completion of model building, the specimen data of art anatomy were imported to VRML to generate a source file. Then, animation playing and frame adjustment were conducted in VRML. After the uploading ended, fine adjustment of scene details was conducted, and digital teaching of art anatomy was completed. Autodesk Maya 3D animation software (produced by Autodesk) was also used.

\subsection{Teaching case}

3D structure design technology includes geometric, physical, and behavioral modeling. (1) Geometric modeling [12]: It may be conducted through toolkit editing, $\mathrm{CAD}$ file importing, and 3D scanning. The core of geometric modeling is the $3 \mathrm{D}$ database. The database includes data files of three levels: high, intermediate, and low. It can accurately achieve equal proportion scaling of the real model and virtual space. Geometric modeling focuses on the description of the appearance of the virtual object, including its shape, surface texture, and illumination. A process chart based on modeling technology is shown in Fig. 4. (2) Physical modeling: The expected motion mode is described by constructing a dynamic system. Generally, the static distant view model is constructed in a digital virtual system. (3) Behavioral modeling: Based on geometric and physical modeling, behavioral modeling needs to process object motion and behavior in order to make the virtual environment realistic.

3DS MAX contains many modeling methods. Different modeling methods may be used according to the shape of the object. The structure design process of 3DS MAX is shown in Fig. 5. 


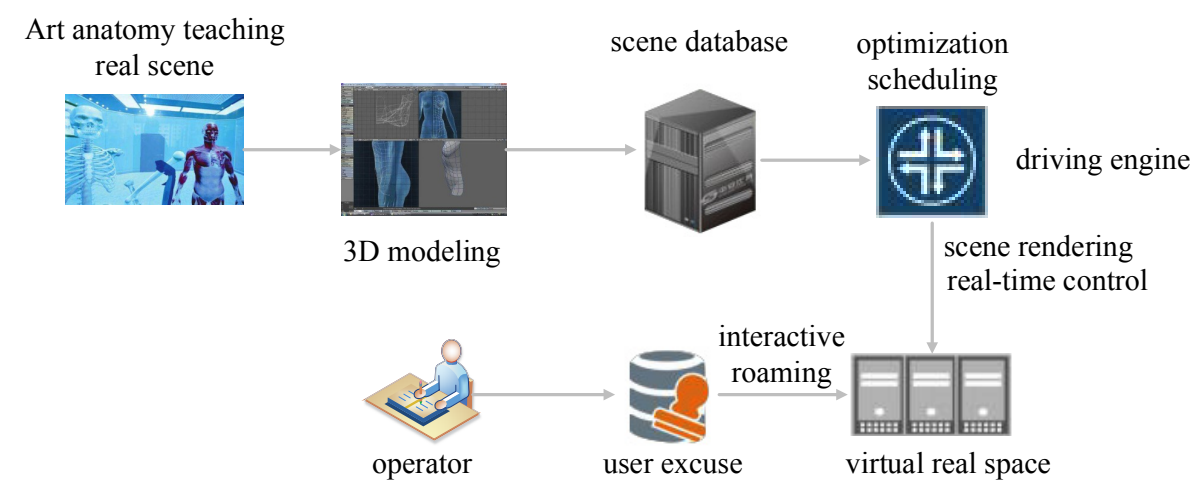

Fig. 4. Process chart of geometric modeling technology

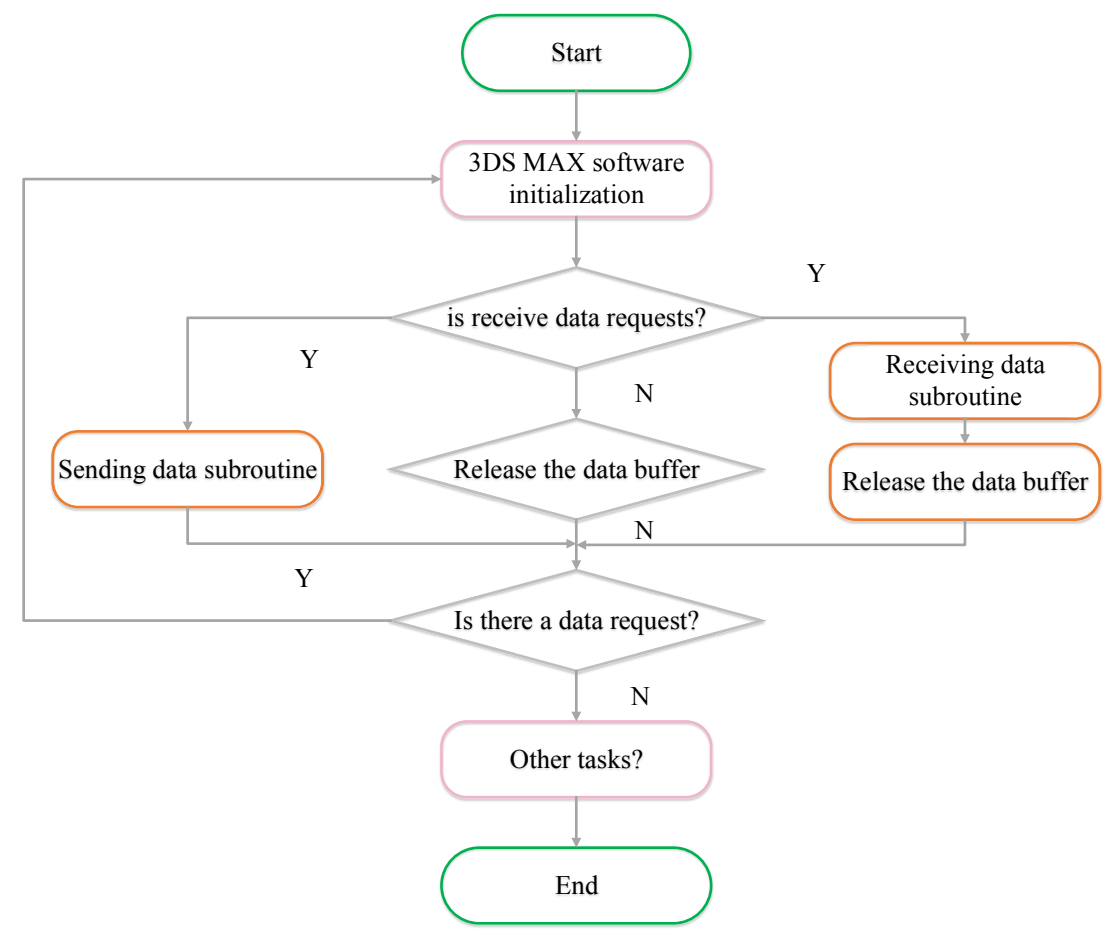

Fig. 5. Structure design process of 3DS MAX

After the structure design of geometric modeling is completed, scene light treatment is required to clearly display the 3D virtual scene. Scene light treatment includes two parts. First, local scene light is used to process the interaction between the objects and light sources, and the correlative dependence of objects is not considered. Second, entire light mainly simulates the reflection and shadow effect between objects to intensify the realism of the scene. In this work, the Gouraud method was used for scene light treatment. Light and shade hue treatment was conducted according to the differ- 
ence in light intensity. In this method, the normal vector of each peak of the virtual object should be known, which can be gained by calculating the mean of normal vectors of adjacent polygons, as shown in Fig. 6.

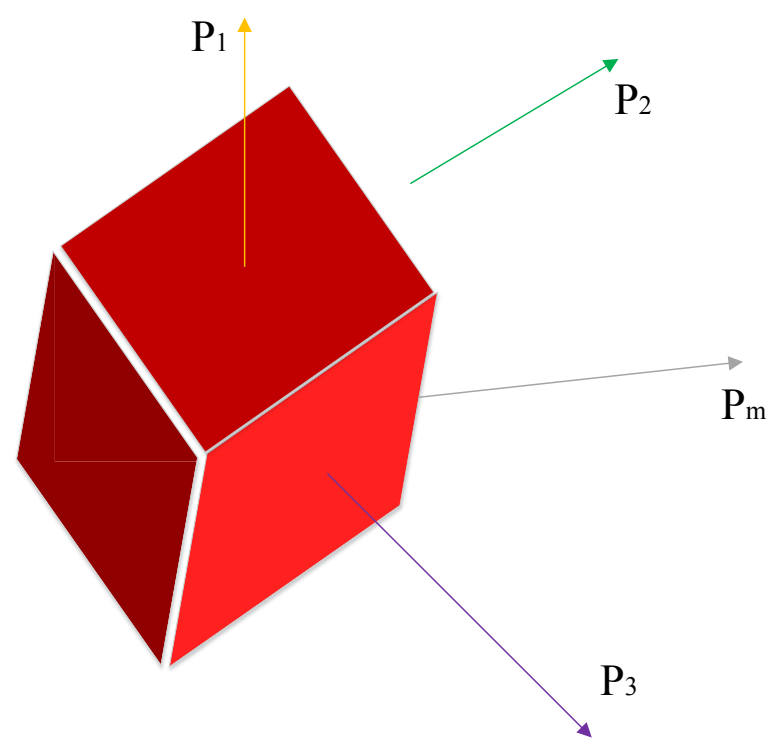

Fig. 6. Scene light treatment diagram

The mean vector $\bar{P}_{M}$ of peak is

$$
\bar{P}_{M}=\sum_{i=1}^{n} \frac{\bar{P}_{i}}{\left|\sum_{i=1}^{n} \bar{P}_{i}\right|}
$$

The light intensity of the peak can be determined according to the normal vector of the peak. Afterward, the light intensity of the side can be calculated. The light intensity of any point on each scanning line can be obtained. On the basis of the structure design of 3DS MAX, the specimen data were imported in VRML according to the route of the virtual camera and light tracing setting. The detailed steps are as follows:

First, the scene was created. The "specimen 1.max" file was established or imported according to the specimen size, namely, "max" files of four side walls (east, south, west, and north) in the corresponding location. Before modeling, detailed picture data for the model to be established was sought as far as possible, and the rough shape was outlined in the mind. Exhaustive understanding of the object is required to establish a fine model. Usually, reference diagrams need to be imported to establish real models. The process is shown in Fig. 7. 


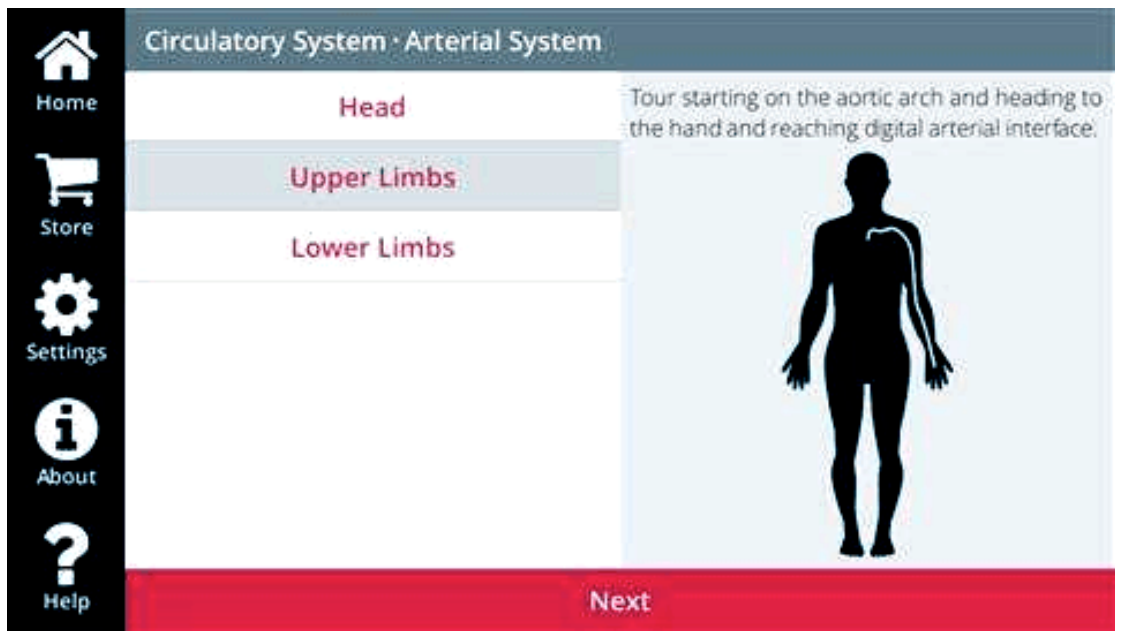

Fig. 7. Reference diagram of anatomy specimen digital import based on digital virtual technology

Second, the setting of the virtual camera was finalized. To acquire appropriate viewpoints in VRML, the virtual camera should be added in the scene to simulate visitors' viewpoints. The camera includes a dynamic camera and several static cameras at different locations and angles. Such a setting can ensure viewpoint dynamics and completeness.

Finally, setting and browsing in VRML were set. The scene generated in 3DS MAX was directly exported to VRML to generate a .wrl source program file. The source program was modified in detail. The scene background, scene transition, and background music were added. (1) Background color addition: the virtual background of VRML included the upper and lower parts, and the XOZ plane served as the boundary. A background image was added to the virtual space of VRML by adjusting the corresponding domain to generate a spatial stereoscopic view. (2) Scene transition: multiple anchor link nodes were added in the digital display. When the visitor clicks a node, scene transition is achieved, and the visitor can be linked to other angles or scenes. The setup of anchor link nodes allows for the transition of different scenes and thus broadens VRML compatibility and endows the digital virtual scene the features of multimedia information. (3) Background music addition: sound can be added in VRML through a sound source and sound emitter. The sound emitter can be created through the sound node. The sound source location is set in the scene.

After background music is added, the rough outline of the specimen side is described on the side view with the EP curve tool. The body part is first done, followed by independent and complex aggregation (several independent organs of the specimen). Then, these are welded. Figs. 8-10 show screenshots of the digital import process of the art anatomy specimen.

The digital art anatomy specimen design and release process described above cannot just train students to master knowledge inquiry, but also lets students acquire a deeper understanding of the knowledge points of art anatomy. 


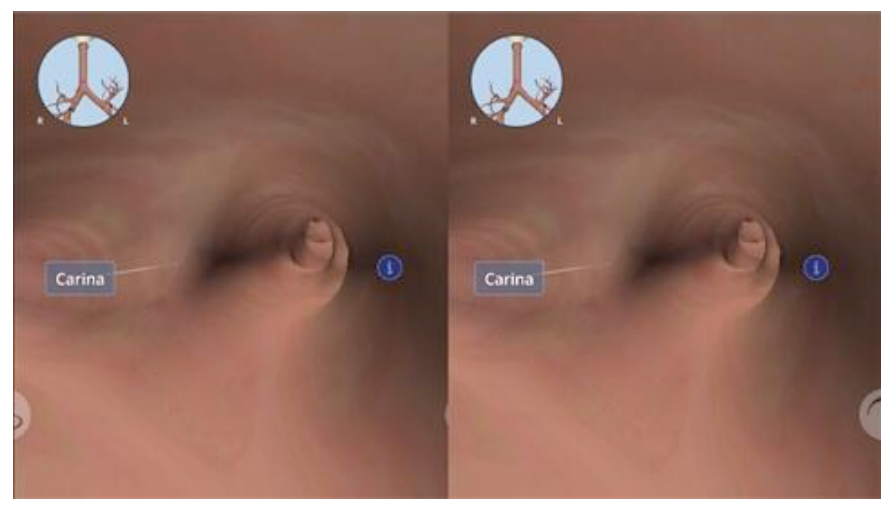

Fig. 8. Screenshot 1 of digital import process of art anatomy specimen

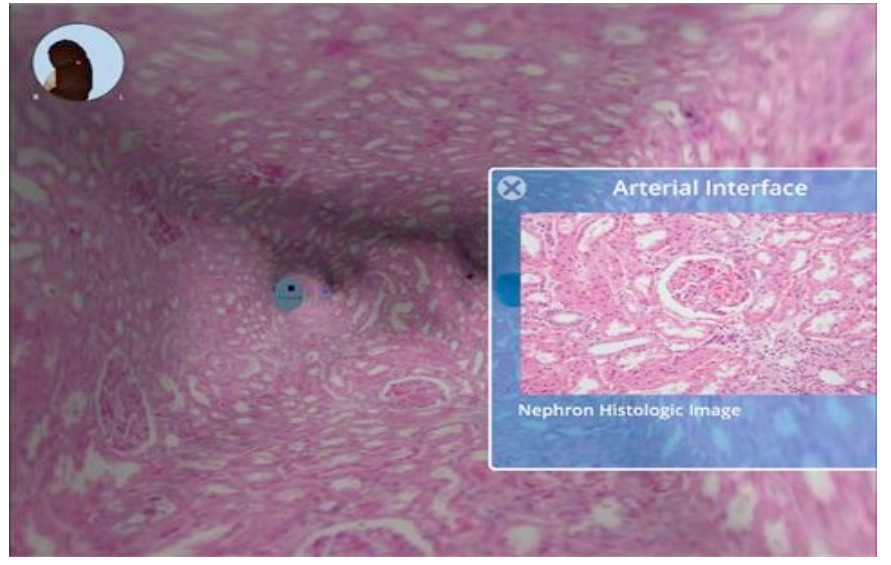

Fig. 9. Screenshot 2 of digital import process of art anatomy specimen

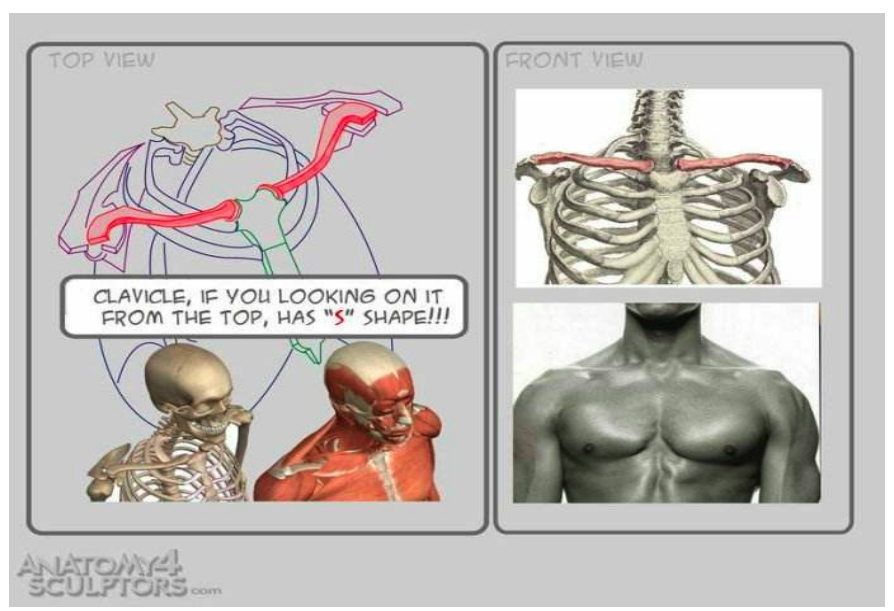

Fig. 10.Screenshot 3 of digital import process of art anatomy specimen 


\subsection{Teaching effect}

To verify the feasibility and effectiveness of the proposed teaching method, the proposed teaching method and the traditional teaching method were applied on two 2016 parallel classes on art anatomy. The students' learning conditions were investigated after the course. The results are shown in Fig. 11. The digital art anatomy teaching method was used for the experimental group, and the traditional teaching method was adopted for the control group. A comparison was made from four aspects: understanding degree for design content, course score, improvement range in the midterm and final test scores, and variance of the final test scores.

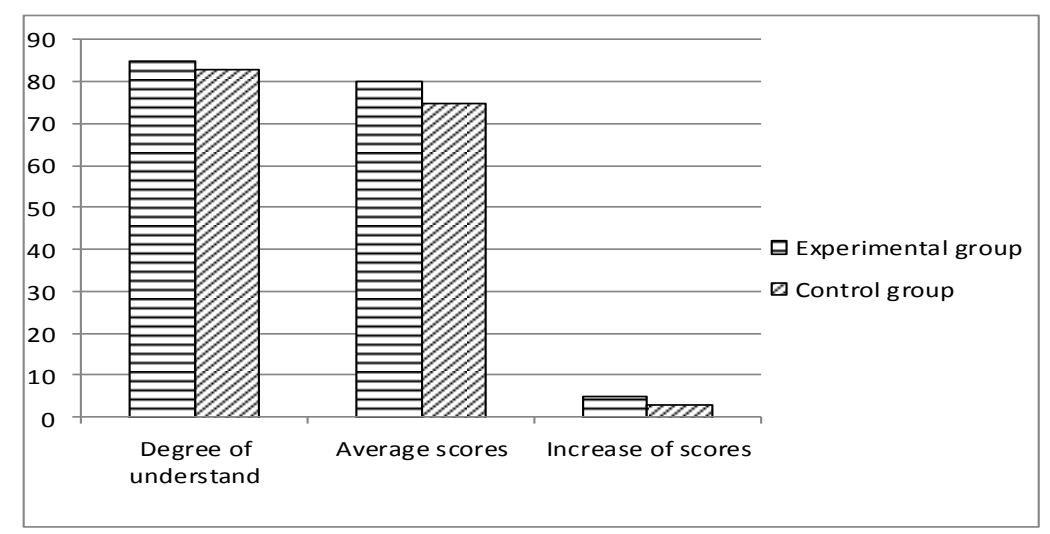

Fig. 11. Teaching effect contrast of experimental group and control group

Fig. 11 shows that the experimental group was superior to the control group. However, due to the immediate effect, the manifestation was not very obvious. In terms of the average score in the final term, the average score of the experimental group was higher than that of the control group. In terms of the average score improvement range, the score of the experimental group was much higher than that of the control group. Therefore, application of the proposed teaching method for a long time exerts an obvious effect.

\section{Conclusions}

Digital virtual technology was combined with art anatomy teaching. The PBL teaching method was applied, and the positive promotion function of the methods in art anatomy teaching was explained in detail. The specimen design of art anatomy was used as an example to introduce the establishment of a 3D model and database of art anatomy specimens via digital virtual technology. 3DS MAX and MAYA software, a strong modeling function, and the vivid scene creation effect of VRML were used to save and record the original state of art anatomy specimens. Moreover, the design process of the PBL teaching method was adopted to evaluate the teaching effect via a questionnaire survey. The following conclusions were drawn. 
1. Digital virtual technology can provide complete, continuous, and fine human 3D anatomic images for art anatomy teaching. 3D visual and virtual human anatomy models can be constructed. With such models, students can observe the human structure from any angle and any profile and can magnify, rotate, and translate the structure to be observed. By carefully observing and clearly understanding the entire image of each structure, students can improve their learning efficiency and reduce teaching resource waste.

2. The PBL teaching method can enlighten and motivate students to learn by themselves and ponder problems. It can motivate them to actively seek knowledge and solve practical problems with the learned knowledge. It contributes to enhancing students' learning interest, exchange with teachers, and learning initiative.

3. With such a teaching method, colleges can indirectly cultivate the ability of art students to grasp and apply software for specimen drawing and meet social requirements for software operation of art students.

\section{References}

[1] Wilkinson, C. Facial reconstruction - anatomical art or artistic anatomy?. Journal of Anatomy, 2010, vol. 216(2), pp. 235. https://doi.org/10.1111/j.1469-7580.2009.01182.x

[2] Hardie, M., Saha, S. Recent experience with problem-based learning in a construction management unit/subject. Journal of the American Chemical Society, 2017, vol. 103(46), pp. 4871-4874.

[3] Piron, L., Cenni, F., Tonin, P., et al. Virtual Reality as an assessment tool for arm motor deficits after brain lesions. Stud Health Technol Inform, 2016, vol. 81, pp. 386-392.

[4] Kurillo, G., Koritnik, T., Bajd, T., et al. Real-time 3D avatars for tele-rehabilitation in virtual reality. Stud Health Technol Inform, 2011, vol. 163, pp. 290-296.

[5] de Faria, J.W., Teixeira, M.J., De, M.S.J.L., et al. Virtual and stereoscopic anatomy: when virtual reality meets medical education. Journal of Neurosurgery, 2016, vol. 125(5), pp. 17. https://doi.org/10.3171/2015.8.JNS141563

[6] Li, Y.F., Yang, M.Y., Shan Y.Y., et al. Application of Three-Dimensional Virtual Digital Visual Human Body in Anatomy Teaching. Anatomy Research, 2012, vol. 34(5), pp. 393394.

[7] Tu, L.G., Liao, B.X., Yang, Y., et al. Application of Three-dimensional Virtual Human Display Platform in Anatomy Teaching of Higher Vocational Medical Technology Specialty. International Journal of Laboratory Medicine, 2017, vol. 38(2), pp. 281-282.

[8] Liu, H.W., Shen, C., Tan, X.G., et al. Explore the use of commonly used memory methods in human anatomy teaching. Progress of Anatomical Sciences, 2012, vol. 18(6), pp. 586587.

[9] Mcfalls, M. Integration of Problem-based Learning and Innovative Technology Into a SelfCare Course. American Journal of Pharmaceutical Education, 2013, vol. 77(6), pp. 117119. https://doi.org/10.5688/ajpe776127

[10] Chen, S., Chen, G., Wang, H. The preliminary study on high efficient acquisition of geophysical data with sparsity constraints. Geophysical Prospecting for Petroleum, 2015, vol. 54(1), pp. 24-35.

[11] Haj-Ali, R., Marom, G., Ben, Z.S., et al. A general three-dimensional parametric geometry of the native aortic valve and root for biomechanical modeling. Journal of Biomechanics, 2012, vol. 45(14), pp. 2392-2397. https://doi.org/10.1016/j.jbiomech.2012.07.017 
Paper-A Teaching Mode for Art Anatomy Based on Digital Virtual Technology

[12] Matsumoto, J.S., Morris, J.M., Foley, T.A., et al. Three-dimensional Physical Modeling: Applications and Experience at Mayo Clinic. Radiographics A Review Publication of the Radiological Society of North America Inc, 2017, vol. 35(7), pp. 1989.

\section{$7 \quad$ Author}

Na Wang is an Assistant teacher in the University of Science and Technology Liaoning, Anshan 114051, China. (3121383141@qq.com).

Article submitted 11 March 2018. Resubmitted 17 June 2018. Final acceptance 23 July 2018. Final version published as submitted by the author. 